\title{
Permeability due to the Increase of Damage in Concrete: From Diffuse to Localized Damage Distributions
}

\author{
Gilles Pijaudier-Cabot ${ }^{1}$; Frédéric Dufour ${ }^{2}$; and Marta Choinska ${ }^{3}$
}

\begin{abstract}
Experimental tests exhibit a strong interaction between material damage and transport properties of concrete. There are at least two asymptotic cases where some theoretical modeling exists: in the case of diffuse cracking, the material permeability should be controlled by damage, e.g., by the decrease of average stiffness due to microcracking. In the case of localized microcracking, and after a macrocrack has formed, permeability should be controlled by a power function of the crack opening (Poiseuille flow). For quasi-brittle materials with evolving microstructure due to mechanical loads, a transition regime on the evolution of permeability between these two asymptotic cases is expected. In this contribution, we define a relationship between permeability and damage that is consistent with the two above configurations. One of the key issues is to relate the crack opening to the state variables in the continuum approach, so that the two asymptotic cases are expressed in the same variable system and can be matched. A simplified approach is used for this purpose. The permeability law is then derived using a mixing formula that weights each asymptotic regime with damage. To illustrate the influence of the matching law on structural response, finite-element simulations of a Brazilian splitting test and a comparison with existing test data are presented.
\end{abstract}

Keywords: Damage; Concrete; Cracking; Permeability; Porous materials.

\section{Introduction}

Transport properties of concrete, like permeability or diffusivity, are particularly important for structures designed for tightness such as prestressed concrete containment vessels. In nuclear vessels for example, tightness to gas is critical during their service life where concrete remains at most microcracked, but also upon minor accidents when macrocracks may appear. Hence, it is important to provide relationships between the amount of cracking in concrete and its permeability that encompass distributed diffuse microcracking and localized macrocracking at the same time. In this paper, we are going to focus interest on the intrinsic permeability related to a solid porous material containing cracks. This parameter depends on the microstructure of the material and disregards the properties of the fluid flowing in the pores (see this classical definition for instance in Choinska et al. (2007a,b).

Experimental test data (Choinska et al. 2007b; Sugiyama et al. 1996), performed on hollow concrete cylinders subjected to compressive loading and at the same time to radial gas flow through

${ }^{1}$ Professor of Civil Engineering, Laboratoire des Fluides Complexes-UPPA/CNRS/Total, Université de Pau et des Pays de l'Adour, Allée du Parc Montaury, 64600 Anglet, France (corresponding author).

${ }^{2}$ Professor of Civil Engineering, Laboratoire 3S-R (Sols, Solides, Structures-Risques)—INPG/UJF/CNRS, ENSE3, BP 5338041 Grenoble Cedex 9, France.

${ }^{3}$ Assistant Professor, GeM, IUT de Saint Nazaire, Département Génie Civil, Ecole Centrale de Nantes/Université de Nantes/CNRS, 58, rue Michel Ange, 44600 Saint-Nazaire, France. their thickness, exhibit several regimes of growth of permeability. The first regime ranges almost up to the peak stress. It is characterized by a slow increase of permeability due to the increase of the density of distributed microcracks. Sometimes, a decrease of permeability may be observed. It is due to the closure of existing microcracks induced by the compressive stress. Nearby the peak stress, a fast increase of the permeability is observed experimentally. It corresponds to the inception of localized cracking. Finally, and for an applied strain, which is larger than several times the peak strain, a third regime may be reached where the specimen contains major through cracks. It is characterized by a decreasing rate of growth of permeability with the applied strain compared to the second regime. Fig. 1 shows these three regimes of growth of the intrinsic permeability of concrete as observed by Choinska et al. (2007b) from experiments where the apparent permeability to gas (nitrogen) was measured. Similar results have been obtained by Torrenti et al. (1999) and Meziani and Skoczylas (1999) in triaxial compression. Experiments investigating the permeability of water provide also similar results, as observed by Kermani (1991) in axial compression, Wang et al. (1997) with splitting tests and Gérard (1996) in uniaxial tension.

The evolution of the permeability of concrete with cracking may be described by two fundamentally different modeling approaches: on one hand, it can be shown theoretically that the permeability of a material, which contains diffuse microcracks is related to the variation of its elastic properties. Dormieux and Kondo (2004) used homogenization techniques and Chatzigeorgiou et al. (2005) analyzed the scaling properties of discrete (lattice) models to derive the same result. In this regime, the permeability can be viewed as a function of a continuum damage based variable that captures the degradation of the elastic stiffness of the material. On the other hand, when microcracking has localized to form a macrocrack, the permeability is a function of the opening, the connectivity and the tortuosity of the crack. Poiseuille flow is the most common assumption [see Dullien (1979)]. 


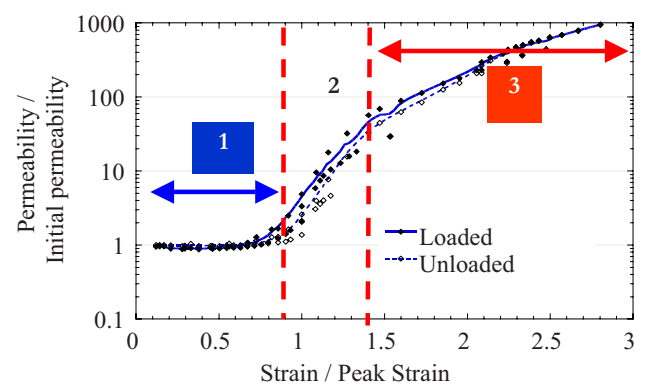

Fig. 1. Evolution of the permeability as a function of the applied strain in a compression test on a hollow cylinder with a radial gas flow

From the viewpoint of permeability, the transition between these two theoretical descriptions is characterized by a percolation threshold, highlighted by Katz and Thompson as reported by Breysse and Gérard (1997). From the mechanical point of view, the same kind of transition is found during the localization of microcracking and their coalescence to form a macrocrack. The experiments reported in Fig. 1 show that this transition may not be sudden, from the hydraulic and mechanical points of view. In compression, microcracking is progressive and the transition toward the formation of macrocrack is smooth, whereas microcracks localize suddenly into a macrocrack in uniaxial tension and the transition is very sharp. The variation of permeability follows the same pace, with a greater range of variation.

Discrete approaches to crack propagation and to fluid flow in fractured media, along with multiscale modeling, provide a possible connection between fluid flow inside existing discontinuities and the overall (macroscopic) governing equations for a porous saturated or unsaturated solid (Réthoré et al. 2007, 2008). If microcracking is accurately characterized, pore network models may also be implemented to describe the influence of micro and macrocracking on the permeability of a porous material [see e.g., the review by Blunt et al. (2002)]. Instead, we have chosen here to follow a simple phenomenological continuum approach. Diffuse microcracking will be described with a damage based formulation. A nonlocal model will be implemented to handle damage localization (macrocracking) due to material strain softening properly. We will then devise a single law governing the variation of the intrinsic permeability as a function of continuum damage that encompasses both distributed damage and localized damage configurations. In doing so, we shall define a consistent relationship matching two extreme configurations: diffuse microcracking described by small distributed damage and macrocracking described by large localized damage.

The equations that describe the mechanical problem are briefly recalled in "Mechanical problem-Continuum damage modeling." The basic reasoning for establishing the matching law is provided in "Permeability matching law." "Computation of a crack opening in continuum damage calculations" is devoted to the computation of the estimate of the crack opening needed in the localized damage configuration. Finally, "Structural simulations" discuss numerical results and comparisons with existing test data in the literature.

\section{Mechanical Problem-Continuum Damage Modeling}

Our purpose is to devise a single relationship between damage and permeability over the entire failure process of the material.
Hence, it is essential to use a setting that allows a proper description of the mechanical response of the material for distributed and localized damage. A possible candidate is the nonlocal damage model (Mazars 1984; Pijaudier-Cabot and Bazant 1987). It offers a simple set of constitutive relations because damage is isotropic. It is clear that damage induced anisotropy would provide some useful information about the anisotropy of the permeability due to directional cracking. Here, directionality of damage and permeability are not considered for the sake of simplicity. Attention is restricted to damage due to tensile loads, which is captured quite satisfactorily by the scalar damage model (see, Mazars and Pijaudier-Cabot 1989; Fichant et al. 1999). The permeability that is investigated corresponds to fluid flow in a plane that is normal to the applied tensile stresses. These restrictions hold whatever the amount of damage experienced by the material.

Damage produces a degradation of the elastic stiffness of the material through a variation of the Young's modulus. The stress strain relation reads

$$
\varepsilon_{i j}=\frac{1+v}{E(1-D)} \sigma_{i j}-\frac{v}{E(1-D)}\left[\sigma_{k k} \delta_{i j}\right] \quad \text { or } \quad \sigma_{i j}=(1-D) C_{i j k l}^{0} \varepsilon_{k l}
$$

$E$ and $\nu=$ Young's modulus and the Poisson's ratio of the undamaged material; $\varepsilon_{i j}$ and $\sigma_{i j}=$ strain and stress components; $C_{i j k l}^{0}$ $=$ initial (elastic) secant stiffness of the material; and $\delta_{i j}$ $=$ Kronecker symbol. The evolution of damage is based on the amount of extension that the material is experiencing during the mechanical loading. In the nonlocal damage model, it is controlled by the average $\bar{\varepsilon}_{\text {eq }}$ of an equivalent strain $\varepsilon_{\text {eq }}$ defined as

$$
\varepsilon_{\mathrm{eq}}=\sqrt{\sum_{i=1}^{3}\left(\left\langle\varepsilon_{i}\right\rangle_{+}\right)^{2}}
$$

where $\langle\cdot\rangle_{+}=$Macauley bracket (positive part of a quantity) and $\varepsilon_{i}=$ principal strains. The equivalent strain is a norm of the positive principal strains. It is a measure of the extensions to which the material is subjected upon loading. Its average is

$$
\bar{\varepsilon}_{\mathrm{eq}}(x)=\frac{\int_{\Omega} \phi(x-s) \varepsilon_{\mathrm{eq}}(s) d \Omega}{\int_{\Omega} \phi(x-s) d \Omega} \text { with } \phi(x-s)=e^{-\left(2\|x-s\| / l_{c}\right)^{2}}
$$

where $\Omega=$ volume of the structure and $\phi(x-s)=$ weight function. $l_{c}=$ internal length of the nonlocal continuum, which is related to the size of the material heterogeneity (Bazant and Pijaudier-Cabot 1989). Note that close to the boundary of the solid, the nonlocal average is adjusted so that upon averaging, a homogeneous local equivalent strain field transforms into the same homogeneous nonlocal field. Close to the boundary, the weight functions are chopped and the weighted average is not the same as for points located far inside the boundaries of the solid. The loading function of damage is

$$
f(Y, \kappa)=Y-\kappa \quad \text { with } Y=\max _{/ t}\left(\bar{\varepsilon}_{\mathrm{eq}}\right)
$$

where $\kappa=$ threshold of damage growth. Initially, $\kappa=Y_{D 0}$. In the course of loading, $\kappa$ assumes the maximum value of the nonlocal equivalent strain ever reached during the loading history 
if $f(Y, \kappa)=0$ and $\dot{f}(Y, \kappa)=0$ then $D=F(Y)$ else $\dot{D}=0$

$F(Y)=$ generic expression of the damage evolution law. To capture the difference of mechanical responses of the material in tension and in compression, the damage variable is split into two parts: $D=\alpha_{t} D_{t}+\alpha_{c} D_{c}$, where $D_{t}$ and $D_{c}$ are the damage variables in tension and compression, respectively. They are combined with the weight coefficients $\alpha_{t}$ and $\alpha_{c}$ [see Pijaudier-Cabot et al. (1991)]. The evolution of damage is provided in an integrated form, as a function of the variable $Y$

$$
\begin{aligned}
& D_{t}=1-\frac{Y_{D 0}\left(1-A_{t}\right)}{Y}-\frac{A_{t}}{e^{\left[B_{t}\left(Y-Y_{D 0}\right)\right]}} \\
& D_{c}=1-\frac{Y_{D 0}\left(1-A_{c}\right)}{Y}-\frac{A_{c}}{e^{\left[B_{c}\left(Y-Y_{D 0}\right)\right]}}
\end{aligned}
$$

Standard values of the model parameters have been provided by Mazars (1984). We will focus attention on the response of the model in tension only. It means that $D_{c}=0, \alpha_{c}=0$, and $\alpha_{t}=1$. The remaining model parameters are the Young's modulus $E$, the Poisson's ratio $v$, the initial damage threshold, constants $A_{t}$ and $B_{t}$, and the internal length $l_{c}$. For more details about this model and the connections with fracture mechanics, (e.g., Mazars and Pijaudier-Cabot 1989,1996).

\section{Permeability Matching Law}

We are going to focus on the intrinsic permeability of the material free of any stress and we will disregard the reversible stress effect on the permeability. In the distributed damage configuration, the permeability of the material may be corrected to account for the reversible stress effect, e.g., according to a function of the applied volumetric confinement stress (e.g., Iscan et al. 2006; Tang et al. 2002). In Poiseuille's expression of the flow rate, the crack opening appears explicitly as we will see next, and it can be corrected to account for the reversible stress effect (crack closure). Before describing how the permeability can be defined over the entire range of variation of damage, let us first discuss the two extreme configurations of diffuse and localized damage.

\section{Diffuse Damage}

To represent the interaction between diffuse damage and permeability at the material level, several proposals exist. Gawin et al. (2003), Bary et al. (2000), and Picandet et al. (2001) have proposed formulas in which the permeability is a power law, or an exponential function of damage. For small enough values of damage (less than 0.15), these formulas are quite equivalent (Jason et al. 2007) and we shall use the relationship due to Picandet et al. (2001) where the intrinsic material permeability is an exponential function of damage

$$
k_{D}=k_{0} \exp \left[(\alpha D)^{\beta}\right]
$$

where $k_{D}$ and $k_{0}=$ current and the initial material permeability, respectively. $\alpha$ and $\beta=$ parameters fitted by the authors to 11.3 and 1.64 , respectively. This empirical relationship is the best fit of the evolution of the permeability measured on (uniaxial) compression specimens after unloading. The permeability has been measured in the direction of the applied load, i.e., perpendicular to the extensions due to Poisson's effect, which cause microcracking in the specimen. It is assumed that it holds similarly in the case of tension damage too. As reported by Picandet and coworkers, Eq. (7) along with the fitted values of the material parameters holds for a variety of concretes (ordinary, high performance, fiber reinforced), and it has been selected so that for standard concrete mixes it can be implemented without any determination of material parameters as a first approximation.

The relationship (7) corresponds to small damage. It holds for damage ranging between 0 and 0.15 , and quickly tends toward infinite values when damage increases. When devising a single relationship between damage and permeability covering the complete range of damage, we will use the Taylor expansion of Eq. (7) to avoid a spurious exponential increase of permeability for large values of damage

$$
k_{D}^{P}=k_{0}\left[1+(\alpha D)^{\beta}+\frac{(\alpha D)^{2 \beta}}{2}+\frac{(\alpha D)^{3 \beta}}{6}\right]
$$

\section{Localized Damage-Crack Opening versus Permeability}

At complete rupture, one or several macrocracks are expected. Hence, fluid flow will be governed by these cracks and Poiseuille's law may be considered. For a fluid flowing between two parallel (rough) plates, the permeability is classically provided by the expression

$$
k_{p}=\zeta \frac{[u]^{2}}{12}
$$

where $[u]=$ crack opening and $\zeta=$ roughness of the crack. Some tortuosity factor may be added too. If we consider a crack of length $L$ in a specimen of cross section $S$ exposed to a gradient of pressure, the total flow rate per unit thickness of specimen (and per unit gradient of pressure) is obtained from Darcy's equation by adding the flow rate through the crack and the flow rate through the undamaged material around the crack. The resulting apparent permeability $k_{\text {ap }}$ is

$$
k_{\text {ap }} \cdot S=\zeta \frac{[u]^{2}}{12} \cdot[u] L+k_{0} S
$$

Note that outside the crack, the permeability is assumed to remain equal to the initial one $k_{0}$. The "crack permeability" $k_{f}$ is the contribution of the crack to the apparent permeability

$$
k_{f}=\zeta \frac{[u]^{3}}{12} \cdot \frac{L}{S}
$$

This permeability corresponds to the second configuration (localized damage) in the relationship between damage and permeability. It is the material permeability that is expected to be reached when damage is close to 1 , at material failure. The difficulty is that $k_{f}$ is a geometry dependent (structural) parameter. It contains the crack length and the cross-section of the material exposed to fluid flow. In the case of multiple periodic cracking, this ratio reduces to the crack spacing (e.g., Gérard 1996).

Within a continuum description of damage, the above expression of the permeability requires the knowledge of the crack location, its length and the possible crack spacing in addition to the crack opening. A more convenient formulation of the contribution of the crack to the apparent permeability can be derived assuming that the crack is replaced by a band of intense damage, as shown in Fig. 2. Let us call $\lambda l_{c}$ the width of this damage band that is equivalent to the macrocrack. It is proportional to the internal 


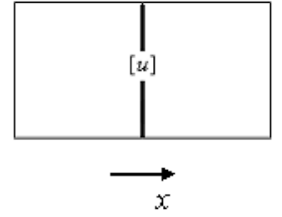

(a)

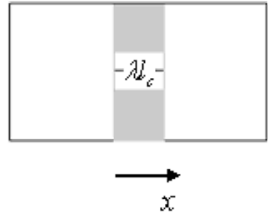

(b)
Fig. 2. (a) Cracked domain (discrete case); (b) damaged domain (continuous case)

length as depicted in (Bazant and Pijaudier-Cabot 1989). For the sake of simplicity, damage is assumed to be constant inside this band and the permeability is denoted as $k_{l}$. The apparent permeability derives now from the flow rate in the band with material permeability $k_{l}$ and in the rest of the specimen with permeability equal to that of the intact material $k_{0}$

$$
k_{\text {ap }} \cdot S=\lambda l_{c} k_{l} \cdot L+k_{0} S
$$

We may now equate Eqs. (10) and (12) and the material permeability $k_{l}$ inside the band is

$$
k_{l}=\frac{\zeta[u]^{3}}{12 \lambda l_{c}}
$$

If this permeability $k_{l}$ is entered at the Gauss point level in a finite element computation, it is expected that the structural hydraulic calculation will provide the apparent permeability defined in Eq. (12). This equivalence requires also that the width of the damage band $\lambda l_{c}$ be small compared to the size of the structure so that the narrow damage band is close to a crack (even though it can be discretized with several finite elements within the width). The remaining difficulty is the calculation of the crack opening. This point will be discussed in "Computation of a crack opening in continuum damage calculations."

\section{Matching Law}

We need now to define a relationship governing the growth of permeability covering the complete range of variation of damage. In addition, this relationship ought to be consistent with the two above extreme configurations of diffuse damage [Eq. (9)] and localized damage [Eq. (13)]. A simple matching law mixing the logarithms of the extreme permeabilities is used

$$
\log (k)=(1-D) \log \left(k_{D}^{p}\right)+D \log \left(k_{l}\right)
$$

This equation satisfies the two following requirements: when damage is close to zero Eq. (9) is recovered, and when damage is close to one Eq. (13) is recovered. Small values of damage correspond to distributed diffuse microcracking while large values of damage correspond to localized macrocracking.

Other combinations can be devised, with the same limits when damage is close to zero and close to one (Choinska et al. 2007a). The difference lays in the relative weights that are given to the two extreme cases. For instance, a linear combination in the form of a standard mixing law $\left(k=(1-D) k_{D}^{p}+D k_{l}\right)$ still satisfies the requirements for small and large damage but at the same time, it lends a greater weight to the small damage regime. The resulting permeability tends toward that of Eq. (13) slower than according to Eq. (14). The variation of the permeability may exhibit a local peak and then decrease, which not very realistic.

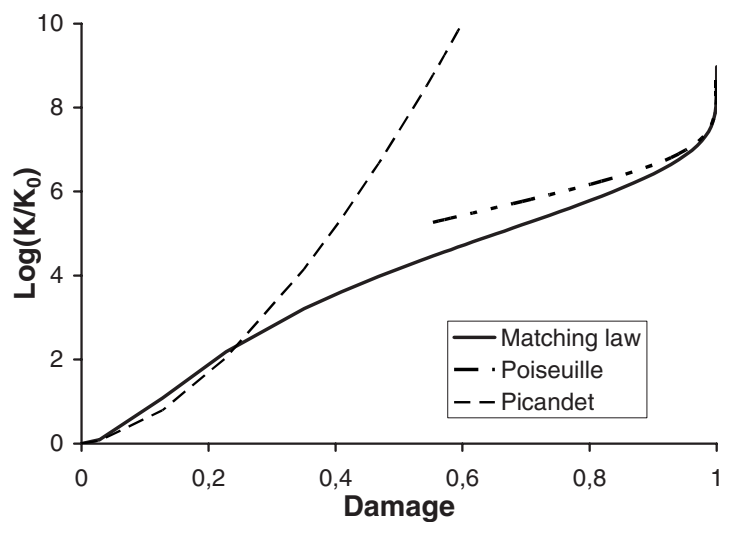

Fig. 3. Evolution of the logarithm of permeability with damage

\section{Computation of a Crack Opening in Continuum Damage Calculations}

The permeability defined in Eq. (14) is a local, point wise quantity, same as the permeability of a continuum described by Darcy's equation. It describes the transition between the two asymptotic configurations of diffuse and localized damage and it is intended to be used in boundary value problems to model the evolution of flow rates across concrete structures upon damage growth. The derivation of a crack opening as a result of a continuum damage mechanics calculation is the remaining step to perform, as it is needed in Eqs. (13) and (14). Once a continuum description of failure has been used to model discontinuous failure, it is not easy to proceed in the reverse direction and to evaluate a crack opening. In recent papers, this problem has been tackled with the assumption that above a given critical value of damage, a displacement discontinuity exists and should be incorporated in the structural model. From this starting point and on, advanced computational models based on an explicit description of the crack (e.g., based on extended finite-element kinematics) provide sound techniques for calculating a displacement discontinuity (Comi et al. 2007; Simone et al. 2003). Following a different approach, Dufour et al. (2008) have devised an approximate direct technique for extracting a discontinuity of displacement from a continuum damage finite element (FE) calculation without the need for enhancing the structural kinematics with displacement discontinuities. Any of these techniques may be used to compute the displacement discontinuity due to crack opening. Nevertheless, we shall devise a simpler and more straightforward estimate of the displacement jump $[u]$.

We assume again that the crack, according to the damage model, is described as a band of intense damage of width $\lambda l_{c}$. The crack opening $[u]$ is equal to the cumulated deformation across (normal to) the damage band. Subsequently, we assume that the crack does not close upon unloading and therefore, the crack opening is computed as if the loading was monotonically increasing. It means that we neglect the reversible displacements upon unloading and that we neglect crack closure (and it yields of course an overestimation of the permeability). We obtain

$$
[u]=\int_{0}^{\lambda l_{c}}\left(Y-Y_{D 0}\right) d x
$$

As the damage distribution in the damaged zone is assumed to be homogeneous, one obtains the crack opening as 
Table 1. Parameters of Mazars' Damage Model

\begin{tabular}{lc}
\hline$E$ & $37.7 \mathrm{GPa}$ \\
$\nu$ & 0.2 \\
$Y_{D 0}$ & $1.10^{-4}$ \\
$A_{t}$ & 1.0 \\
$B_{t}$ & 15,600 \\
$l_{c}$ & $20 \mathrm{~mm}$ \\
\hline
\end{tabular}

$$
[u]=\left(Y-Y_{D 0}\right) \lambda l_{c}
$$

By substitution of Eqs. (5) and (6) in Eq. (16), the crack opening may be represented as a function of the damage

$$
[u]=\left(F^{-1}(D)-Y_{D 0}\right) \lambda l_{c}
$$

It should be recalled that this derivation remains based on simple-not to say simplistic_-assumptions. The equivalence between the damage band and the crack assumes that the entire deformation of the band is converted into crack opening, which is an overestimation of the crack opening. Some part of this deformation corresponds to material damage around the crack that is not part of crack opening. Recall also that we investigate the permeability of the unloaded material. Upon unloading the crack may probably close although in practice it does not close completely due to dilatancy during the failure process and crack roughness. The closure of the crack is not accounted for here.

For the sake of illustration, let us consider the material parameters given in Table 1 and let us compute the evolution of material permeability as described by the matching law in Eq. (14). The internal length $l_{c}$ is arbitrary chosen equal to $0.02 \mathrm{~m}$, while $\lambda$ is equal to 2 . The initial permeability $k_{0}$ considered in the simulations is taken equal to $10^{-17} \mathrm{~m}^{2}$ and $\zeta=1$. The growth of permeability with damage and with the variable that controls the growth of damage is shown in Figs. 3 and 4. The matching law provides a correct representation of the permeability for damage ranging between 0 and 0.15 , as well as for strong damage where it tends toward the permeability given according to Poiseuille's law.

\section{Structural Simulations}

To illustrate the matching permeability law on a structural configuration, we consider concrete disks loaded either according to the splitting test or to the BIPEDE experiment (Gérard 1996) (see Fig. 5). In this latter one, a disk made of mortar is glued to steel

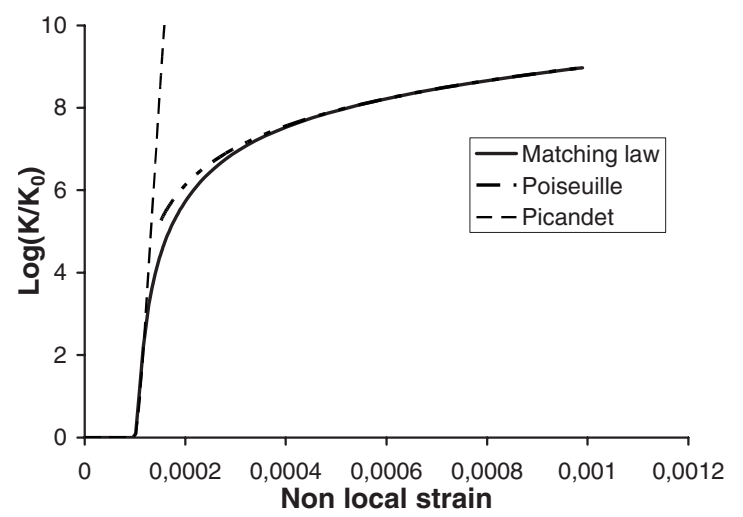

Fig. 4. Evolution of the logarithm of permeability with the nonlocal equivalent strain

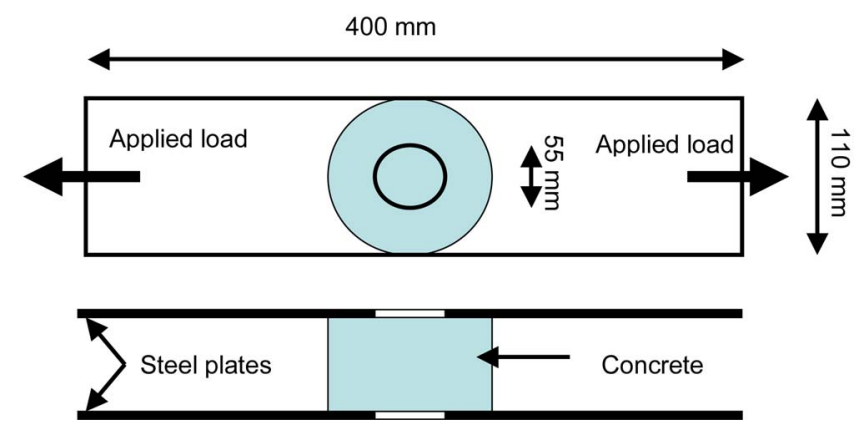

Fig. 5. BIPEDE test geometry [after Gérard (1996)]

plates. Tension is applied to the plates and it is transmitted to the disk, in which one or two transverse cracks appear. At the same time water flows through holes in the steel plates and through the disk. The apparent permeability (to water) is measured in the course of loading and cracking of the disk. In the present comparisons, we shall consider the tests in which a single crack develops. Note that in the splitting test and in the BIPEDE test, the two distributions of damage are quite similar.

\section{Mechanical Problem-Brazilian Splitting Test}

The cylindrical specimen is loaded along a diametral plane by means of steel bearing plates, as shown in Fig. 6. The steel bearing plates are arbitrary modeled by elastic plates, with a high Young's modulus $(E=300 \mathrm{GPa})$ and a Poisson's ratio equal to that of concrete to avoid a local confinement effect on concrete. The set of parameters of Mazars' damage model (see Table 1) represents ordinary concrete behavior. The internal length is equal to $0.02 \mathrm{~m}$.

Numerical simulations are performed with four-node quadrangle elements. Due to double symmetry, the computation domain consists in one quarter of a specimen. A plane stress calculation is performed. Damage distributions at peak and at failure when damage has propagated over the specimen diameter are depicted in Figs. 7(a and b). One can observe that damage develops in a band of a limited width (governed by the internal length).

\section{Evolution of Apparent Permeability}

For every damage state, a material permeability is computed at each Gauss point according to the matching law [Eqs. (14) and (17)] and to the relationship due to Picandet alone, which stands

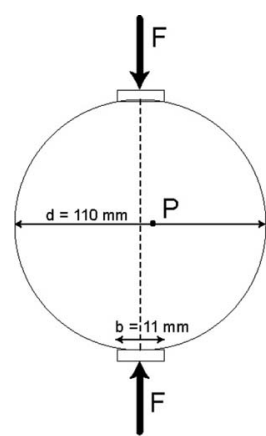

(a)

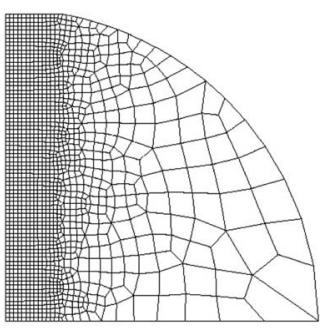

(b)
Fig. 6. Brazilian splitting test: (a) problem statement; (b) FE mesh 


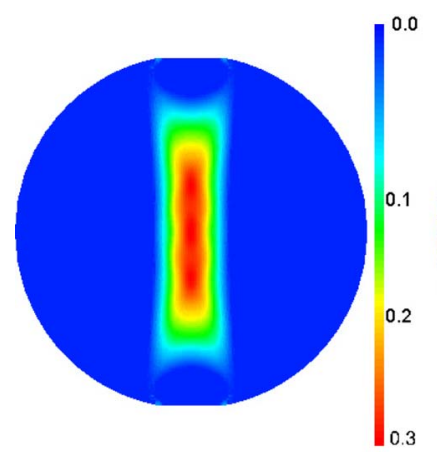

(a)

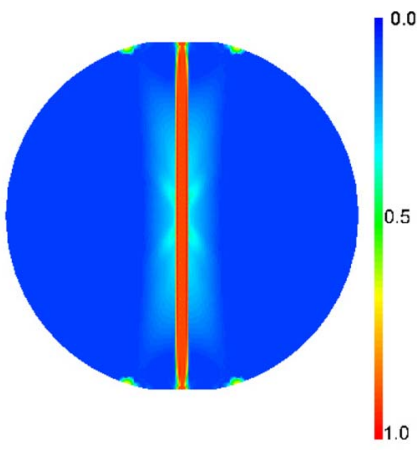

(b)
Fig. 7. Damage distributions at (a) peak; (b) at the last loading step

in the case of small damage only [Eq. (7)]. Then, a structural permeability is determined by averaging the permeability over the surface of the specimen exposed to fluid flow. Results in Fig. 8 show the evolution of the average permeability with the crack opening displacement (COD) to peak COD ratio. With the matching formula in Eq. (14) the shape of the curve is similar to that in Fig. 1, and it is also similar to the results by Wang et al. (1997). The comparison is qualitative but it is clear that the relationship between damage and permeability corresponding to diffuse damage [Eq. (7)] cannot be extended to capture the overall permeability when localized damage is observed. When this relationship is implemented alone, the fact that damage may localize into a narrow band does not provide the relative decrease of the rate of growth of permeability observed experimentally. Fig. 8 shows that Eq. (7) yields an overestimate of the apparent permeability of the disk. The structural (average) permeability increases by 20 orders of magnitude almost for the COD at peak. It is unrealistic and far above the range of variation of five to seven orders of magnitude that is observed experimentally. The same realistic range is observed according to the matching law in Eq. (14).

Quantitative comparisons with experiments can be provided with the data obtained in the BIPEDE experiment for which the mechanical damage model used here as been calibrated. Gérard reported experimental data concerning the apparent structural permeability to water, same as in Eqs. (10) and (12) indexed by the tensile strain in concrete during the experiment. This tensile strain is homogeneous and it is computed assuming that damage devel-

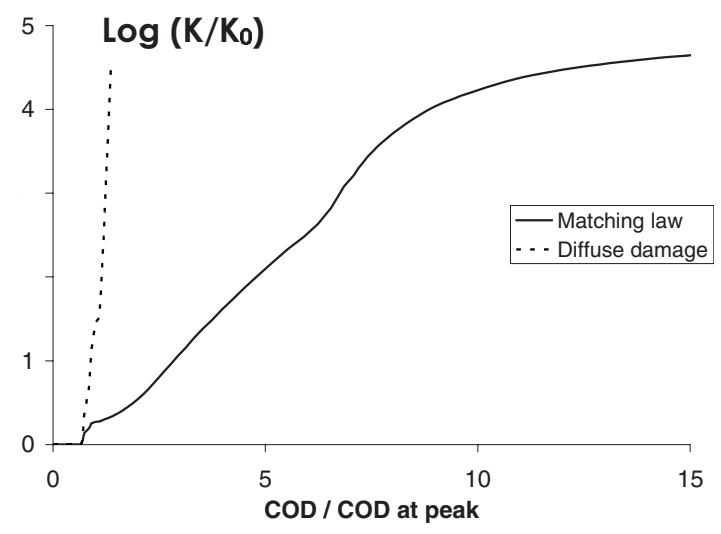

Fig. 8. Evolution of the logarithm of the structural apparent permeability (normalized by the initial permeability) with the COD to peak COD ratio
Table 2. Parameters of Mazars' Damage Model Used by Gérard (1996)

\begin{tabular}{lc}
\hline$E$ & $35 \mathrm{GPa}$ \\
$v$ & 0.2 \\
$Y_{D 0}$ & $1.510^{-4}$ \\
$A_{t}$ & 1.0 \\
$B_{t}$ & 6,000 \\
$l_{c}$ & $30 \mathrm{~mm}$ \\
\hline
\end{tabular}

ops into a band of width $\lambda l_{c}$ and of length equal to the diameter of the specimen. This strain profile (with a constant strain) is related to the strain profile computed according to the nonlocal damage model according to an equivalence of energy dissipation due to damage [see Bazant and Pijaudier-Cabot (1989)]. In this equivalence, the local strain within the band is equal to the peak nonlocal strain. The local strain corresponds also to the average strain measured experimentally during the tests as reported by Gérard (1996). Given this strain, damage and the equivalent crack opening are computed from Eqs. (5), (6), and (17).

Gérard (1996) provided the calibration of the damage model used in the present study. The model parameters correspond to concrete D65. They are provided in Table 2. The internal length is three times the maximum aggregate size. The model parameters in Eq. (7) are those defined in "Permeability matching law" and $\zeta=0.1$ in Eq. (13). $\lambda$ is equal to 1.89 , same as in Bazant and Pijaudier-Cabot (1989). The initial apparent permeability $K_{0}$ is $5.10^{-12} \mathrm{~m} / \mathrm{s}$ and the dynamic viscosity of water is $\eta$ $=10^{-3} \mathrm{~kg} / \mathrm{m} / \mathrm{s}$.

Fig. 9 shows the comparison between the experiments and the present matching law. The agreement with experimental data are quite satisfactory. In the figure, we have also plotted the simulation by Gérard, which takes into account the opening of the crack explicitly and uses Eq. (9) only in the damage band, the permeability in the rest of the specimen being kept equal to the initial one [Eq. (10)]. The apparent permeability computed according to the matching law is quite close to that computed by Gérard on the basis of Poiseuille's law only. As opposed to Gérard formula, our approach does not rely on a crack system known in advance. The distribution of damage is the result of the mechanical computation, in which the relationship governing the permeability for any value of damage is inserted point wise. Note also that for small to moderate damage a formulation that would rely on Eq. (10), on Poiseuille law only, would not be consistent from a theoretical point of view until a macrocrack is formed for which a crack opening displacement exists.

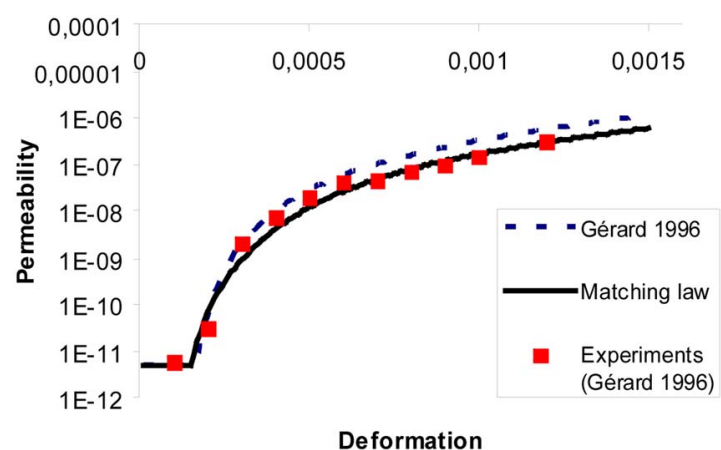

Fig. 9. Evolution of the permeability to water with the strain in concrete in the "BIPEDE" test 


\section{Conclusions}

In this contribution, a formula describing the evolution of permeability with damage has been proposed. It matches consistently two extreme configurations: in the first one, permeability is an exponential function of distributed diffuse damage, while in the second one it is governed by crack opening. By analytical variables substitution, we have associated the crack opening with the variable that governs damage in a nonlocal integral damage model and afterwards we have related this state variable with damage to arrive to an expression where the permeability is controlled by the variation of material damage solely. An extensive comparison of the predictions of permeability with experimental data on split cylinders is in progress, but initial comparisons provide qualitative and quantitative results that are quite consistent with existing experimental data.

\section{Acknowledgments}

Financial support from the Agence Nationale de la Recherche under the project "Contifiss" is gratefully acknowledged.

\section{References}

Bary, B., Bournazel, J. P., and Bourdarot, E. (2000). "Poro-damage approach applied to hydro-mechanical fracture analysis of concrete." $J$. Eng. Mech., 126, 937-943.

Bazant, Z. P., and Pijaudier-Cabot, G. (1989). "Measurement of the characteristic length of nonlocal continuum." J. Eng. Mech., 115, 755767.

Blunt, M., Jackson, M. D., Piri, M., and Valvatne, P. H. (2002). "Detailed physics, predictive capabilities, and macroscopic consequences for pore-network models of multiphase flow." Adv. Water Resour., 25, 1069-1089.

Breysse, D., and Gérard, B. (1997). "Modelling of permeability in cement-based materials: Part 1-Uncracked medium." Cem. Concr. Res., 27, 761-775.

Chatzigeorgiou, G., Picandet, V., Khelidj, A., and Pijaudier-Cabot, G. (2005). "Coupling between progressive damage and permeability of concrete: Analysis with a discrete model." Int. J. Numer. Analyt. Meth. Geomech., 29, 1005-1018.

Choinska, M., Dufour, F., and Pijaudier-Cabot, G. (2007a). Matching permeability law from diffuse damage to discontinuous crack opening, new trends in fracture mechanics of concrete, A. Carpinteri, P. Gambarova, G. Ferro, and G. Plizzari, eds., Taylor and Francis, London, 541-548.

Choinska, M., Khelidj, A., Chatzigeorgiou, G., and Pijaudier-Cabot, G. (2007b). "Effects and interactions of temperature and stress-level related damage on permeability of concrete." Cem. Concr. Res., 37, 79-88.

Comi, C., Mariani, S., and Perego, U. (2007). “An extended Fe strategy for transition from continuum damage to Mode I cohesive crack propagation.” Int. J. Numer. Analyt. Meth. Geomech., 31, 213-238.

Dormieux, L., and Kondo, D. (2004). "Approche micromécanique du couplage perméabilité-endommagement." C.R. Mecanique, 332, 135140.

Dufour, F., Pijaudier-Cabot, G., Choinska, M., and Huerta, A. (2009).
"Extraction of crack opening from a continuous approach using regularised damage models." Comput. Concr., 10, 375-388.

Dullien, F. A. L. (1979). Porous media-Fluid transport and pore structure, Academic, San Diego.

Fichant, S., La Borderie, C., and Pijaudier-Cabot, G. (1999). "Isotropic and anisotropic descriptions of damage in concrete structures." Mech. Cohesive-Frict. Mater, 4, 339-359.

Gawin, D., Pesavento, F., and Schrefler, B. A. (2003). "Modelling of hygro-thermal behaviour of concrete at high temperature with thermochemical and mechanical material degradation." Comput. Methods Appl. Mech. Eng., 192, 1731-1771.

Gérard, B. (1996). "Contribution of the mechanical, chemical, and transport couplings in the long-term behavior of radioactive waste repository structures." Ph.D. dissertation, Univ. Laval and Ecole Normale Supérieure de Cachan.

Iscan, A. G., Kok, M. V., and Bagci, A. S. (2006). "Estimation of permeability and rock mechanical properties of limestone reservoir rock under stress conditions by strain gauge." J. Pet. Sci. Eng., 53, 13-24.

Jason, L., Pijaudier-Cabot, G., Ghavamian, S., and Huerta, A. (2007). "Hydraulic behaviour of a representative structural volume for containment buildings." Nucl. Eng. Des., 237, 1259-1274.

Kermani, A. (1991). "Stressed concrete-Permeability of stressed concrete." Build. Res. Inf., 19, 360-366.

Mazars, J. (1984). "Application de la mécanique de l'endommagement au comportement non linéaire et à la rupture de béton de structure." $\mathrm{Ph} . \mathrm{D}$. thesis, Université Pierre et Marie Curie, Paris.

Mazars, J., and Pijaudier-Cabot, G. (1989). "Continuum damage theory: Application to concrete." J. Eng. Mech., 115, 345-365.

Mazars, J., and Pijaudier-Cabot, G. (1996). "From damage to fracture mechanics and conversely: A combined approach." Int. J. Solids Struct., 33, 3327-3342.

Meziani, H., and Skoczylas, F. (1999). "Experimental study of the mechanical behavior of a mortar and of its permeability under deviatoric loading." Mater. Struct., 32, 403-409.

Picandet, V., Khelidj, A., and Bastian, G. (2001). "Effect of axial compressive damage on gas permeability of ordinary and highperformance concrete." Cem. Concr. Res., 31, 1525-1532.

Pijaudier-Cabot, G., and Bazant, Z. P. (1987). "Nonlocal damage theory." J. Eng. Mech., 113, 1512-1533.

Pijaudier-Cabot, G., Mazars, J., and Pulikowski, J. (1991). "Steelconcrete bond analysis with nonlocal continuous damage." J. Struct. Eng., 117, 862-882.

Réthoré, J., de Borst, R., and Abellan, M. A. (2007). "A two scale approach for fluid flow in fractured porous media." Int. J. Numer. Methods Eng., 71, 780-800.

Réthoré, J., de Borst, R., and Abellan, M. A. (2008). "A two-scale model for fluid flow in an unsaturated porous medium with cohesive cracks." Comput. Mech., 42, 227-238.

Simone, A., Wells, G. N., and Sluys, L. J. (2003). "From continuous to discontinuous failure in a gradient enhanced continuum damage model." Comput. Methods Appl. Mech. Eng., 192, 4581-4607.

Sugiyama, T., Bremner, T. W., and Holm, T. A. (1996). "Effect of stress on gas permeability in concrete." ACI Mater. J., 93, 443-450.

Tang, C. A., Tham, L. G., Lee, P. K. K., Yang, T. H., and Li, L. C. (2002). "Coupled analysis of flow, stress, and damage (FSD) in rock failure." Int. J. Rock Mech. Min. Sci., 39, 477-489.

Torrenti, J. M., Didry, O., Ollivier, J. P., and Plas, F. (1999). La dégradation des bétons, couplage fissuration-dégradation chimique, Communications en mécanique, Hermes Pubs, Paris.

Wang, K., Jansen, D. C., Shah, S. P., and Karr, A. F. (1997). "Permeability study of cracked concrete." Cem. Concr. Res., 27, 381-393. 